\title{
Passification-Based Adaptive Control : Robustness Issues *
}

\author{
Dimitri Peaucelle $^{\dagger}$, Alexander Fradkov ${ }^{\ddagger}$, Boris Andrievsky ${ }^{\ddagger}$ \\ $\dagger$ LAAS-CNRS \\ 7, av. du colonel Roche, 31077 Toulouse, FRANCE \\ Email: peaucelle@laas.fr \\ $\ddagger$ Institute for Problems of Mechanical Engineering of RAS \\ 61 Bolshoy av. V.O., St Petersburg, 199178, RUSSIA \\ Email: $\{$ alf , bandri $\} @$ control.ipme.ru
}

September 19, 2005

\begin{abstract}
Passivity is a widely used concept in Control Theory having lead to many significant results. Here we concentrate on one characteristic of passivity, namely passification-based adaptive control. This concept applies to MIMO systems for which exists a combination of outputs that renders the open-loop system hyperminimum-phase. Under such assumptions, the system may be passified by both high-gain static output-feedback and by a particular adaptive control algorithm. This last control law is modified here to guarantee its coefficients to be bounded. The contribution of the paper is to investigate its robustness with respect to parametric uncertainty. Time response characteristics are illustrated on examples including realistic situations with noisy output and saturated input. Theoretical results are formulated as Linear Matrix Inequalities and can hence be readily solved with Semi-Definite Programming solvers.
\end{abstract}

Keywords: Passivity, Robustness, Adaptive Control.

\section{INTRODUCTION}

One of a variety of adaptive control approaches is the so-called passification based adaptive control $(P B A C)[4,5,13,1,8]$ providing efficient design procedures and simple controller structures. Passification (sometimes called passivation) is understood as finding a state or output feedback rendering the closed loop system passive. Among

*This work is supported by CNRS-RAS research cooperation program No. 16394 and Complex Program of the Presidium of RAS \#19 "Control of mechanical systems", project 1.4. 
applications areas of passification based adaptive control are process control [2], flight control [3,7], irrigation systems [15]. Although robust properties with respect to parametric uncertainties of PBAC were demonstrated in different problems by means of simulation, no rigorous results were obtained by now. The aim of this paper is to give constructive methods for robust PBAC and to show that a modification of the PBAC allows to constrain the convergence of time-varying controller coefficients. This last feature being particularly efficient when considering perturbed outputs.

In the next section we first give some preliminaries and recall the $G$-passivity concept that extends passivity to non-square systems. The third section is then devoted to the proposed Bounded Passification-Based Adaptive Controller (BPBAC) and robustness with respect to parametric uncertainty is proved under parameter-dependent static output-feedback $G$-passivity conditions. At this stage the BPBAC needs to be applied to go along with a methodology for finding appropriate matrices $G$ and a procedure for proving the existence of parameter-dependent controllers. These two problems are solved in sections 4 and 5 respectively. Section 6 is then devoted to simulations of the BPBAC and the section 7 concludes the paper.

\section{Preliminaries}

Notations: $\mathrm{R}^{m \times n}$ and $\mathrm{C}^{m \times n}$ are the sets of $m$-by- $n$ real and complex matrices respectively. $A^{T}$ is the transpose of the matrix $A$ and $A^{*}$ is its transpose conjugate. $A^{\perp}$ is a full rank matrix whose columns span the null-space of $A$. 1 and 0 are respectively the identity and the zero matrices of appropriate dimensions. For Hermitian matrices, $A>(\geq) B$ if and only if $A-B$ is positive (semi) definite. Define $\phi_{h}(k): C \rightarrow C$ the dead-zone function parametrized by $h>0$, such that:

$$
\phi_{h}(k)= \begin{cases}0 & (|k| \leq h) \\ \left(1-\frac{h}{|k|}\right) k & (|k| \geq h)\end{cases}
$$

and if $k$ is a vector or a matrix, $\phi_{h}$ applies elements-wize. Before stating the main problem of the paper, a technical lemma is given for this dead-zone operator.

Lemma 1 Let $f \in C$ such that $|f| \leq h$, then for all $k \in C$ the inequality $(k-$ $f)^{*} \phi_{h}(k) \geq 0$ holds.

Proof : If $|k| \leq h$ then $\phi(k)=0$, the inequality is trivial. If $|k| \geq h$ then $(k-f)^{*} \phi(k)$ is the scalar product of two vectors in the complex plane as shown on the figure below

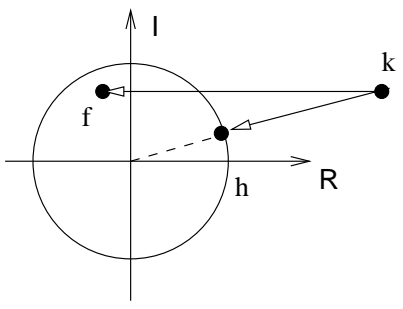


and is necessarily nonnegative.

Consider a non-linear uncertain system

$$
\Sigma(\Delta):\left\{\begin{array}{l}
\dot{x}=f(x, \Delta)+g(x) u \\
y=h(x)
\end{array}\right.
$$

where $x=x(t) \in \mathrm{C}^{n}, u=u(t) \in \mathrm{C}^{m}, y=y(t) \in \mathrm{C}^{l}$ are state, input and output vectors respectively, $\Delta$ is an uncertain constant matrix known to belong to a set $\Delta$ and $f, g$ and $h$ are smooth functions.

Let $G$ be a prespecified $m \times l$-matrix. Extending the definitions of [6] to uncertain systems, the system (1) is called robustly strictly G-passive if for any $\Delta \in \Delta$ there exists a nonnegative scalar function $V(x, \Delta)$ (storage function) and a scalar function $\rho(x, \Delta)>0$ such that

$$
\begin{aligned}
& V(x(t), \Delta) \leq V(x(0), \Delta) \\
& \quad+\int_{0}^{t}\left[u(\theta)^{*} G y(\theta)-\rho(x(\theta), \Delta)\right] d \theta
\end{aligned}
$$

holds for all solutions of the system (1).

Obviously, if $l=m$ and $G=1$ is identity matrix, then $G$-passivity coincides with conventional passivity property. Moreover, for a given $G$, the modified system $G \Sigma$ where the output $y(t)$ is replaced by $G y(t)$ is strictly passive if and only if the original system $\Sigma$ is strictly $G$-passive. But $G$-passification of $\Sigma$ (i.e. finding $K$ such that the closed-loop with $u=K(y)+v$ is passive) is not equivalent to passification of $G \Sigma$. In case of static feedback $(u=K y+v)$, these two problems coincide only under the assumption of finding a square matrix $F$ such that $K=F G$. This "squaring down" procedure changes the number of unknowns which may be important to preserve, e.g. for adaptive control, where reducing the number of adjustable parameters may decrease transient performance of adaptive systems, see [1]. To summarize, $G$-passification of $\Sigma$ is not equivalent to passification of $G \Sigma$, thus justifying the terminology.

\section{Bounded Passification-Based Adaptive Control}

The paper focuses on the control of uncertain LTI systems

$$
\left\{\begin{array}{l}
\dot{x}=A(\Delta) x+B u \\
y=C x
\end{array}\right.
$$

where $B$ and $C$ are assumed to be full rank and where $\Delta$ is a constant unknown parameter that belongs to a given set $\Delta$.

The central result to be studied in the paper is now formulated.

Theorem 1 Assume $G \in \mathrm{C}^{m \times l}$ be a given matrix, the following (i) condition implies (ii):

(i) There exists a parameter-dependent $m \times l$ matrix $F(\Delta)$ with all elements bounded by $h$ in modulus (i.e. $\phi_{h}(F(\Delta))=0$ ) such that system (3) with the output feedback

$$
u(t)=F(\Delta) y(t)+v(t)
$$


is robustly strictly G-passive.

(ii) For any l-uplet of Hermitian positive definite matrices $\Gamma_{i} \in \mathrm{C}^{m \times m}$, the choice

$$
\begin{aligned}
& u(t)=K(t) y(t) \\
& \dot{K}_{i}(t)=-y_{i}^{*}(t) \Gamma_{i} G y(t)-\Gamma_{i} \phi_{h}\left(K_{i}(t)\right)
\end{aligned}
$$

where $K_{i}$ are the $l$ columns of $K$ and $y_{i}$ the $l$ scalar elements if $y$, is a timevarying output-feedback that renders system (3) robustly strictly G-passive.

Proof: Assume $F(\Delta)$ robustly strictly $G$-passifies the system. According to [6], this is equivalent for all values of $\Delta \in \Delta$ to the existence of a quadratic storage function $V(x, \Delta)=x^{*} H(\Delta) x$ and a positive scalar $\rho(\Delta)>0$ fulfilling the matrix inequalities

$$
\begin{gathered}
H(\Delta)=H^{*}(\Delta)>0 \quad: \quad H(\Delta) B=C^{*} G^{*} \\
H(\Delta) A(\Delta, F)+A^{*}(\Delta, F) H(\Delta)<-2 \rho(\Delta) 1
\end{gathered}
$$

where $A(\Delta, F)=A(\Delta)+B F(\Delta) C$ is the closed-loop dynamics matrix. Let any $l$ uplet of $m \times m$ Hermitian positive definite matrices $\Gamma_{i}>0$ and let the output-feedback law (5). Consider the following storage function

$$
\begin{aligned}
& V(x, K, \Delta)=\frac{1}{2} x^{*} H(\Delta) x \\
& \quad+\frac{1}{2} \sum_{i=1}^{l}\left(K_{i}-F_{i}(\Delta)\right)^{*} \Gamma_{i}^{-1}\left(K_{i}-F_{i}(\Delta)\right)
\end{aligned}
$$

where $F_{i}(\Delta)$ are the columns of $F(\Delta)$. Along the trajectories of (3) with the control law (5) the derivatives of $V(x, K, \Delta)$ write

$$
\begin{aligned}
\dot{V}(x, K, \Delta)= & x^{*} H(\Delta)(A(\Delta) x+B K y+B v) \\
& +\sum_{i=1}^{l}\left(K_{i}-F_{i}(\Delta)\right)^{*} \Gamma_{i}^{-1} \dot{K}_{i} .
\end{aligned}
$$

Add and subtract $x^{*} H(\Delta) B F(\Delta) y$ in the equation to get

$$
\begin{aligned}
\dot{V}(x, K, \Delta)= & x^{*} H(\Delta)(A(\Delta) x+B F(\Delta) y+B v) \\
& +y^{*}(K-F(\Delta))^{*} B^{*} H(\Delta) x \\
& +\sum_{i=1}^{l}\left(K_{i}-F_{i}(\Delta)\right)^{*} \Gamma_{i}^{-1} \dot{K}_{i} .
\end{aligned}
$$

Develop the following product

$$
y^{*}(K-F(\Delta))^{*}=\sum_{i=1}^{l}\left(K_{i}-F_{i}(\Delta)\right)^{*} y_{i}^{*},
$$

recall that (6) implies $B^{*} H(\Delta) x=G C x=G y$ and take into account the dynamics of $K_{i}(t)$ to get

$$
\begin{aligned}
& \dot{V}(x, K, \Delta) \leq-\rho(\Delta)\|x\|^{2}+v^{*} G y \\
& \quad-\sum_{i=1}^{l}\left(K_{i}-F_{i}(\Delta)\right)^{*} \phi_{h}\left(K_{i}\right) .
\end{aligned}
$$

Moreover denoting $k_{i j}$ and $f_{i j}$ the scalar components of $K$ and $F(\Delta)$ respectively, the last term of (8) writes as

$$
\sum_{i=1, j=1}^{l, m}\left(k_{i j}-f_{i j}\right)^{*} \phi_{h}\left(k_{i j}\right) .
$$


Due to Lemma 1 all these elements are positive and hence one gets

$$
\dot{V}(x, K, \Delta) \leq-\rho(\Delta)\|x\|^{2}+v^{*} G y
$$

For a zero input $v=0$, the derivative of $V(x, K, \Delta)$ is definite negative for all trajectories and this holds for any $\Delta \in \Delta$. The closed-loop is robustly globally asymptotically stable. Taking the integral over time of the derivative of the storage function, one gets

$$
\begin{aligned}
& V(x(t), K(t), \Delta) \leq V(x(0), K(0), \Delta) \\
& +\int_{0}^{t}\left[v(\theta)^{*} G y(\theta)-\rho(\Delta)\|x(\theta)\|^{*}\right] d \theta
\end{aligned}
$$

Which is (2) for the closed-loop.

\section{Remarks:}

The time-varying control (5) is called the Bounded Passification-Based Adaptive Controller (BPBAC). The values of the gain $K(t)$ are automatically tuned given the measures $y(t)$, it adapts whatever the values of the uncertain parameters $\Delta$ and thanks to the dead-zone, the controller gains are constrained to converge in a bounded set.

The result formulated in Theorem 1 makes BPBAC attractive for robust outputfeedback but it needs

- A methodology for finding a $G$ matrix such that the system may be $G$-passified via static-output feedback;

- A result for assessing the existence of a parameter-dependent static output-feedback law with bounded coefficients such that (i) holds for a given set of uncertainties $\Delta$

- A demonstration that the non-linear algorithm properties are not destroyed by perturbations on the system and in particular to noise on the measurements.

These aspects are treated in the following sections.

\section{BMI design of $G$ matrices}

In this section we aim at designing $G$ matrices such that the system may be robustly $G$-passified via static output-feedback. It appears that this problem is the major difficulty of the overall problem we consider. In particular, it may not be approximated by a convex optimization under LMI constraints. Hence, for more simplicity we shall consider the $G$-design problem without robustness issues at first. The next section will give robustness analysis conditions for a given $G$.

Let the nominal system obtained for zero uncertainty:

$$
\left\{\begin{array}{l}
\dot{x}=A(0) x+B u=A x+B u \\
y=C x
\end{array}\right.
$$

In [6, Corollary 3] is proved that if there exists a static output-feedback gain that $G$-passifies the system then the choice $K=-k G$, for a sufficently large value of $k$, 
is also a $G$-passifying gain. This result is quite common in passivity context and is known as high-gain control. For our purpose, it allows to simplify the search of $G$. Indeed $G$-passifiability conditions (6) write for $K=-k G$ :

$$
\begin{gathered}
H=H^{*}>0: \quad H B=C^{*} G^{*} \\
H A+A^{*} H<2 k C^{*} G^{*} G C
\end{gathered}
$$

This problem is non-convex and if $k$ is chosen very large a priori it writes as Bilinear Matrix Inequalities (BMIs). It may therefore be solved using the PenBMI solver [9, 10]. Of course, since the problem is not convex there is no guarantee to finding $G$ even when it exists. However, as tested on several examples, PenBMI does succeed efficiently.

The design procedure we have adopted for $G$ is

- Choose a large value of $k$;

- Choose an upper bound on $H$ (we took $\bar{h}=1$ ) for scaling the solutions;

- Declare in YALMIP the following BMI problem where $H, G$ and $t$ are the variables

$$
\begin{gathered}
\bar{h} 1>H>0, H B=C^{*} G^{*}, t>-1 \\
H A+A^{*} H-k C^{*} G^{*} G C<t 1
\end{gathered}
$$

- Minimize $t$ using PenBMI, if it returns $t<0$, the procedure succeeded.

This design procedure is applied to the following numerical example that is used all along the paper.

Example: Consider the linearized fourth-order model of lateral dynamics for an autonomous aircraft including model of actuator dynamics, presented in [7]. The nominal model is defined for a medium value of the flight altitude $h=5 \mathrm{~km}$. The measured plant output $y(t)$ is a vector of the yaw angle $\psi(t)$, yaw angular rate $r(t)$ and the rudder deflection angle $\delta_{r}(t): y(t)=\left[\psi, r, \delta_{r}\right]^{*}$. The control input of the plant is the rudder servo command signal, i.e. $n=4, m=1, l=3$.

Parameters of the state-space model (3) in this case are as follows:

$$
A=\left[\begin{array}{cccc}
0 & 1 & 0 & 0 \\
0 & 0 & 1 & 0 \\
0 & 12 & -0.6 & 5.0 \\
0 & 0 & 0 & -20
\end{array}\right], B=\left[\begin{array}{c}
0 \\
0 \\
0 \\
20
\end{array}\right] C=\left[\begin{array}{cccc}
1 & 2 & 0 & 0 \\
0 & 1 & 2 & 0 \\
0 & 0 & 0 & 1
\end{array}\right]
$$

We have tested the numerical procedure with two choices of $k$ :

$$
k_{1}=10^{3} \quad, \quad k_{2}=10^{5}
$$

to get the following two admissible values of $G$

$$
\begin{aligned}
G_{1 \text { PenBMI }} & =10^{-2}\left[\begin{array}{lll}
4.5404 & 2.8436 & 1.7107
\end{array}\right] \\
G_{2 \text { PenBMI }} & =10^{-3}\left[\begin{array}{lll}
8.4000 & 5.4505 & 3.0961
\end{array}\right]
\end{aligned}
$$

and a simple analysis step shows that the scaled and rounded values

$$
G_{1}=\left[\begin{array}{lll}
4 & 3 & 2
\end{array}\right], G_{2}=\left[\begin{array}{lll}
8 & 5 & 3
\end{array}\right]
$$

are admissible as well. These are used next. 


\section{Finding admissible uncertainty sets}

At this stage to apply the results of Theorem 1 it is needed to characterize uncertainty sets such that condition (i) holds. To do so let us detail the adopted uncertain modeling: $A(\Delta)$ is assumed rational with respect to $\Delta$

$$
A(\Delta)=A+B_{\Delta} \Delta\left(1-D_{\Delta} \Delta\right)^{-1} C_{\Delta}
$$

where $\Delta$ is a constant norm-bounded unstructured uncertainty that belongs to the set

$$
\Delta_{\rho}=\left\{\Delta \in \mathrm{C}^{m_{\Delta} \times l_{\Delta}}: \Delta^{*} \Delta \leq \rho^{2} 1\right\} .
$$

The uncertainty model is said to be a Linear Fractional Transform (LFT), build as the feedback connection of the uncertain matrix $\left(w_{\Delta}=\Delta z_{\Delta}\right)$ with the linear system

$$
\left\{\begin{array}{l}
\dot{x}=A x+B_{\Delta} w_{\Delta}+B u \\
z_{\Delta}=C_{\Delta} x+D_{\Delta} w_{\Delta} \\
y=C x
\end{array}\right.
$$

The LFT is assumed to be well-posed, that is $\left(1-D_{\Delta} \Delta\right)$ is non-singular for all admissible uncertainties $\Delta \in \Delta$ and $A(\Delta)$ is in a bounded set.

For such uncertain systems, based on results on hyper minimum-phaseness and passivilty [5, 6], an LMI test for robust $G$-passifiability was recently published in [12]. Slightly modified to take into account uncertain sets with $\rho \neq 1$ it writes as follows.

Theorem 2 [12] If there exists a solution $H \in \mathrm{C}^{n \times n}, F \in \mathrm{C}^{m \times l}$ to the LMI constraints

$$
\begin{aligned}
& H>0, H B=C^{*} G^{*} \\
& {\left[\begin{array}{cc}
H A+A^{*} H+C^{*}\left(G^{*} F+F^{*} G\right) C & H B_{\Delta} \\
B_{\Delta}^{*} H & -1
\end{array}\right]} \\
& +\rho^{2}\left[\begin{array}{c}
C_{\Delta}^{*} \\
D_{\Delta}^{*}
\end{array}\right]\left[\begin{array}{ll}
C_{\Delta} & D_{\Delta}
\end{array}\right]<0
\end{aligned}
$$

then the uncertain system is robustly strictly G-passified via static output-feedback $u(t)=F y(t)+v(t)$ for all uncertainties $\Delta \in \boldsymbol{\Delta}_{\rho}$.

Adding to the LMIs (13) the convex constraint that the modulus of all elements of $F$ must be bounded by $h$, gives a convex semi-definitie programing problem for finding $F$ such that (i) in Theorem 1 . Moreover, as $\rho^{2}$ enters linearly in the LMI it may be maximized in order to optimize the size of the admissible uncertainty set.

Note that this result gives a static output-feedback that does not depend on the uncertain parameters. It therefore fulfills more restrictive conditions than those expected for (i). But, an important point is that the actual set of admissible uncertainties that satisfies the condition may not be of the norm-bounded type. Moreover, Theorem 2 being possibly conservative the largest admissible norm-bounded set may not be obtained. Nevertheless, the actual set of admissible uncertainties can eventually be approximated via unions of norm-bounded set. If for each set a static output-feedback gain such that (i) can be proved to exist, the result being the existence of a parameter-dependent static 
output-feedback of the type (4) for the union of sets. This feature is now illustrated on the numerical example.

Example: Assume the model is affected by uncertainties $\Delta$ on the flight altitude. The nominal model matrix $A$ corresponds to the flight altitude of $5 \mathrm{~km}$, therefore we look for admissible uncertainties such that $\Delta>-5$. The parameters of the uncertain modeling are as follows:

$$
\begin{gathered}
B_{\Delta}=\left[\begin{array}{llll}
0 & 0 & 0.2 & 0
\end{array}\right]^{T}, D_{\Delta}=0 \\
C_{\Delta}=\left[\begin{array}{llll}
0 & -7.5 & 0.7 & -4.5
\end{array}\right] .
\end{gathered}
$$

Note that for this data some coefficients of the matrix $A(\Delta)$ vary in an order of magnitude when $\Delta$ varies from -5 to $5 \mathrm{~km}$.

Apply the LMIs of Theorem 2 to this example with the constraint that the controller parameters should all be bounded by $h=10$. In order to approximate the set of admissible uncertainties by norm-bounded sets the maximization of $\rho$ is performed with different values of the nominal model. To do so, Theorem 2 is applied with $A$ replaced by $A\left(\Delta_{0}\right)$ where $\Delta_{0}$ is the altitude around which the norm-bounded set is searched.

Two series of experiments are performed, one for each matrix $G_{1}$ and $G_{2}$ computed in the previous section. The results are given in Tables 1 and 2. Obtained admissible uncertainty sets $\Delta \subset \mathrm{C}$ are plotted in Figures 1 and 2.

Table 1: Results of Theorem 2 for $G_{1}$

\begin{tabular}{|c|c|c|c|c|}
\hline$\Delta_{0}$ & $\rho$ & \multicolumn{3}{|c|}{$F$} \\
\hline-5 & 1.3752 & -9.1563 & -6.9588 & -10 \\
\hline-3 & 1.3945 & -9.2994 & -8.3421 & -10 \\
\hline-1 & 1.4135 & -9.8670 & -9.6170 & -10 \\
\hline 1 & 1.2914 & -10 & $\begin{array}{ll}-10 & -8\end{array}$ & 591 \\
\hline 3 & 0.9202 & -10 & $\begin{array}{ll}-10 & -3\end{array}$ & 329 \\
\hline 4 & 0.2950 & -5.9472 & -6.5527 & 0.2024 \\
\hline 4.2 & 0.0976 & -1.8782 & -2.2142 & 0.7575 \\
\hline
\end{tabular}

Table 2: Results of Theorem 2 for $G_{2}$

\begin{tabular}{|c|c|c|}
\hline$\Delta_{0}$ & $\rho$ & $F$ \\
\hline-5 & 1.6702 & $\begin{array}{lll}-9.4123 & -6.6019 & -10 \\
\end{array}$ \\
\hline-2 & 1.7097 & $\left.\begin{array}{lll}-9.7243 & -9.1112 & -10\end{array}\right]$ \\
\hline 1 & 1.5601 & $-10 \quad-10 \quad-7.8995$ \\
\hline 3 & 1.1596 & $\begin{array}{lll}-10 & -10 & -3.9229\end{array}$ \\
\hline 4 & 0.6095 & $-10 \quad-9.6843 \quad-0.4888$ \\
\hline 4.6 & 0.0213 & $-0.5449 \quad-0.6375 \quad 0.8501$ \\
\hline
\end{tabular}

From these experiments is obtained that there exists a bounded parameter dependent static output-feedback such that the system is robustly $G_{1}$-passsifiable for all real valued uncertainties in $[-54.2976]$ and $G_{2}$-passifiable for all uncertainties in 


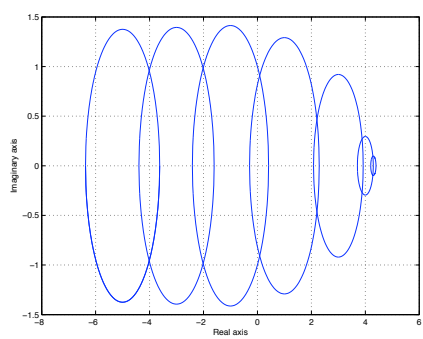

Figure 1: Unions of admissible uncertain sets for $G_{1}$

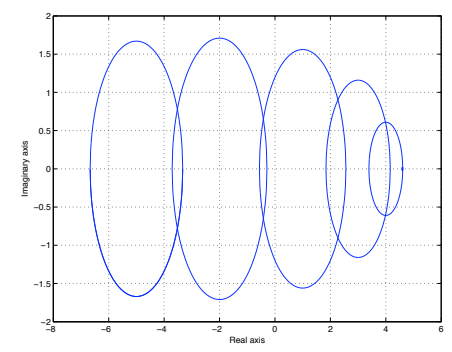

Figure 2: Unions of admissible uncertain sets for $G_{2}$

[ -54.6213$]$. For these sets of uncertainties the BPBAC (5) asymptotically stabilizes the system and renders if $G$-passive.

\section{Simulations of the BPBAC}

Tests are performed for $G_{2}$ that gives the largest admissible set of flight altitudes. The BPBAC is tested taking all $\Gamma_{i}=1$ and initial conditions

$$
x(0)=\left(\begin{array}{llll}
1 & 0 & 0 & 0
\end{array}\right)^{T}, K(0)=\left[\begin{array}{lll}
0 & 0 & 0
\end{array}\right] .
$$

First experiments are made for $\Delta=0$ and $\Delta=4.6$ and time histories of both the output $y(t)$ and the control gain $k(t)$ are plotted on Figures 3 and 4 . As expected the stabilization of the system becomes more critical as the uncertainty grows. Other simulations for $\Delta=4.62$ show that convergence to zero takes about 1000 seconds.

Second experiments are performed for a more realistic situation with noise on the measurements $(y(t)=C x(t)+n(t))$ and saturation on the inputs $(u(t)$ is saturated between -20 and +20$)$. The time responses for $\Delta=3$ are plotted on Figure 5 . Notice that after 10 seconds the controller parameters have converged to a stabilizing static output feedback

$$
K(10)=\left[\begin{array}{lll}
-10.0511 & -7.1310 & -2.8870
\end{array}\right]
$$



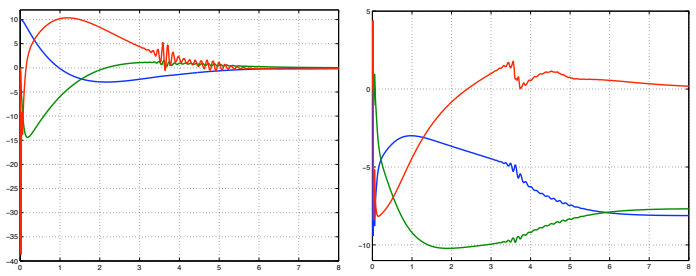

Figure 3: $y(t)$ and $k(t)$ histories for $\Delta=0$
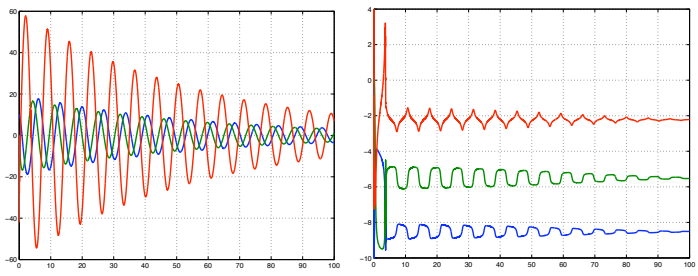

Figure 4: $y(t)$ and $k(t)$ histories for $\Delta=4.6$

such that the eigenvalues of $A(\Delta=3)+B K(10) C$ are all negative:

$-68.3711,-8.5017,-0.5235 \pm 0.7221 i$.
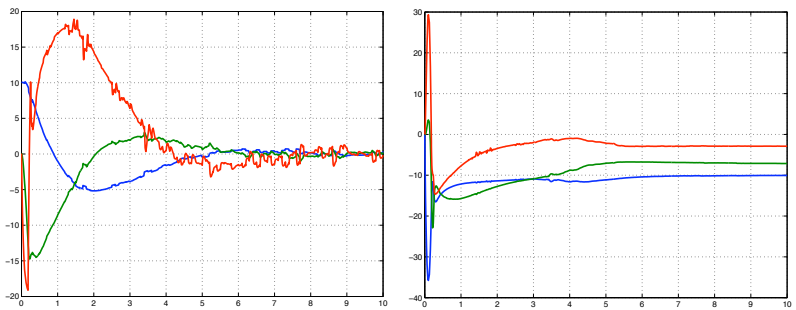

Figure 5: $y(t)$ and $k(t)$ histories with noisy measurements

Notice in Figure 5 that one of the coefficients converges close to the limit value $h=10$. This is due to the dead-zone operator introduced in the BPBAC. Without this element (that is taking $h=\infty$ ), the controller gains would diverge under the influence of the perturbations $n(t)$.

All numerical calculations are performed in the MATLAB environment. YALMIP [11] is used to enter LMIs and BMIs. Semi-definite programming problems are solved with SeDuMi [14] and BMI problems are solved with PenBMI [9, 10]. 


\section{Conclusions}

Based of passification framework a new adaptive controller is proposed. It is proved to have both parametric robustness and convergence to bounded values properties. Conditions on uncertain parameters for robustness to hold are formulated in terms of the existence of a parameter-dependent static controller. A by-product of our results is to build explicitly such controller using LMI tools. Compared to such controller that needs to measure the uncertain parameters for being implemented, the BPAC adapts automatically whatever the uncertainties.

Although robustness with respect to external perturbations such as measurement noise is illustrated on examples, a prospective work will be devoted to proving this property theoretically.

\section{References}

[1] B.R. Andrievsky, A.N. Churilov, and A.L. Fradkov. Feedback KalmanYakubovich lemma and its applications to adaptive control. In IEEE Conference on Decision and Control, pages 4537-4542, June 1996.

[2] B.R. Andrievsky and A.L. Fradkov. Adaptative controllers with implicit reference models based on Kalman-Yakubovich lemma. In IEEE Conference on Control Application, pages 1171-1174, Glasgow, September 1994.

[3] B.R. Andrievsky and A.L. Fradkov. Combined adaptative flight control system. In 5th Intern. ESA Conf. Spacecraft Guidance, Navigation and Control Systems, pages 299-302, Frascati, Italy, October 2002. (ESA-516, Feb. 2003).

[4] A.L. Fradkov. Adaptative stabilization of an linear dynamic plant. Autom. Remote Contr., 35(12):1960-1966, 1974.

[5] A.L. Fradkov. Quadratic Lyapunov functions in the adaptive stabilization problem of a linear dynamic plant. Siberian Math. J., 2:341-348, 1976.

[6] A.L. Fradkov. Passification of non-square linear systems and feedback Yakubovich-Kalman-Popov lemma. European J. of Control, 6:573-582, 2003.

[7] A.L. Fradkov and B.R. Andrievsky. Shunting method for control of homing missiles with uncertain parameters. In IFAC Symposium on Automatic Control in Aerospace, volume 2, pages 33-38, St. Petersburg, Russia, June 2004.

[8] A.L. Fradkov, I.V. Miroshnik, and V.O. Nikiforov. Nonlinear and Adaptative Control of Complex Systems. Kluwer Academic Publishers, Dordrekht, 1999.

[9] M. Kočvara and M. Stingl. PENNON - a code for convex nonlinear and semidefinite programming. Opt. Methods and Software, 18(3):317-333, 2003.

[10] M. Kočvara and M. Stingl. PENBMI, version 2.0, 2004. see www . penopt . com for a free developer version. 
[11] J. Löfberg. YALMIP3, 2004. http:// control.ee.ethz.ch/ joloef/ yalmip.msql.

[12] D. Peaucelle, A.L. Fradkov, and B.R. Andrievsky. Robust G-passification via static output feedback - LMI results. In 16th IFAC World Congress, Prague, Czech Republic, July 2005.

[13] M.M. Seron, D.J. Hill, and A.L. Fradkov. Adaptive passification of nonlinear systems. In IEEE Conference on Decision and Control, pages 190-195, December 1994.

[14] J.F. Sturm. Using SeDuMi 1.02, a MATLAB toolbox for optimization over symmetric cones. Optimization Methods and Software, 11-12:625-653, 1999. URL: fewcal.kub.nl/sturm/software/sedumi.html.

[15] A.M. Tsykunov. Adaptative Control of Retarded Systems. Nauka, Moscow (In Russian), 1984. 